PROCEEDINGS OF THE

AMERICAN MATHEMATICAL SOCIETY

Volume 135, Number 11, November 2007, Pages 3537-3546

S 0002-9939(07)08851-X

Article electronically published on July 3, 2007

\title{
REGIONS OF POSITIVITY FOR POLYHARMONIC GREEN FUNCTIONS IN ARBITRARY DOMAINS
}

\author{
HANS-CHRISTOPH GRUNAU AND GUIDO SWEERS \\ (Communicated by Walter Craig) \\ Dedicated to Prof. J. Serrin on the occasion of his 80th birthday
}

\begin{abstract}
The Green function for the biharmonic operator on bounded domains with zero Dirichlet boundary conditions is in general not of fixed sign. However, by extending an idea of $\mathrm{Z}$. Nehari, we are able to identify regions of positivity for Green functions of polyharmonic operators. In particular, the biharmonic Green function is considered in all space dimensions. As a consequence we see that the negative part of any such Green function is somehow small compared with the singular positive part.
\end{abstract}

\section{INTRODUCTION AND MAIN RESULTS}

We are interested in positivity preserving properties of the biharmonic Dirichlet boundary value problem

$$
\begin{cases}\Delta^{2} u=f & \text { in } \Omega, \\ u=|\nabla u|=0 & \text { on } \partial \Omega,\end{cases}
$$

i.e. in the question, which was raised by Hadamard Ha1, Ha2, whether positive data $f \geq 0$ always yield positive solutions $u \geq 0$. It is by now well known that the answer is affirmative, e.g. in balls [B], small perturbations of the two dimensional disk GS1, GS2, S and even in some nonconvex two dimensional domains DS2. However, in general, this property does not hold true as was shown by many counterexamples $[\mathrm{C}, \mathrm{CD}, \mathrm{CG}, \mathrm{D}, \mathrm{Ga}, \mathrm{O}, \mathrm{ST}$. The question mentioned is closely related to the positivity of the corresponding Green function. Let us assume that the bounded domain $\Omega \subset \mathbb{R}^{n}$ is $C^{4, \alpha}$-smooth. Then, by [ADN], for every $f \in C^{0, \alpha}(\bar{\Omega})$ one has a unique classical solution $u \in C^{4, \alpha}(\bar{\Omega})$. That means that the Green function $G_{\Omega}:=G_{\Delta^{2}, \Omega}$ corresponding to the Dirichlet problem (11) exists and that the solution can be given by

$$
u(x)=\int_{\Omega} G_{\Omega}(x, y) f(y) d y .
$$

The question of Hadamard may now be rephrased as whether one has

$$
G_{\Omega}(x, y) \geq 0 \quad \text { or even } \quad G_{\Omega}(x, y)>0 .
$$

Received by the editors February 13, 2006 and, in revised form, July 7, 2006.

2000 Mathematics Subject Classification. Primary 35J65, 35B50, 35J40.

(C)2007 American Mathematical Society Reverts to public domain 28 years from publication 
As explained above, this question in general cannot be answered in the affirmative. However, one observes in numerical examples that the negative part of $G_{\Omega}$ seems to be very small when compared with its positive part. So, here we pose the question to identify subsets

$$
\mathcal{P} \subset \Omega \times \Omega \backslash\{(x, x)\}
$$

such that

$$
\forall(x, y) \in \mathcal{P}: \quad G_{\Omega}(x, y)>0 .
$$

If such a set $\mathcal{P}$ can be identified to be relatively large, this would show that the negative part of the Green function is indeed relatively small. This question is not only of interest in its own, but may play a crucial role in treating nonlinear equations.

A first step to identify positivity sets $\mathcal{P}$ was done by Nehari $[\mathrm{N}]$ in space dimensions $n=2$ and $n=3$. His result will be described after Theorem 1 below. Related problems were treated from different points of view by Malyshev [M] and Dall'Acqua, Meister and the second author DMS.

We write

$$
d(x)=\operatorname{dist}(x, \partial \Omega)
$$

Developing Nehari's idea, we are able to prove the following result.

Theorem 1. Let $n \geq 4$. Then there exists a constant $\delta_{n}>0$, which depends only on the dimension $n$, such that the following holds true.

Assume $\Omega \subset \mathbb{R}^{n}$ to be a $C^{4, \alpha}$-smooth bounded domain and let $G_{\Omega}:=G_{\Delta^{2}, \Omega}$ denote the Green function for the biharmonic operator under Dirichlet boundary conditions. If

$$
|x-y|<\delta_{n} \max \{d(x), d(y)\}
$$

then we have

$$
G_{\Omega}(x, y)>0
$$

For the constant $\delta_{n}$, one may achieve that

$$
\delta_{4} \geq 0.59, \quad \delta_{n} \geq 0.6 \text { for } n \geq 5
$$

and that

$$
\lim _{n \rightarrow \infty} \delta_{n}=\frac{\sqrt{5}-1}{2} \approx 0.618
$$

Such a result was proved by Nehari $[\mathrm{N}]$ in the three dimensional case $n=3$ with a constant $\delta_{3}=4-2 \sqrt{3}=0.535898384 \ldots$. For two dimensional domains, only a much more restricted statement seems to be available, where also the maximal distance of $x, y$ to boundary points of $\partial \Omega$ is involved; see also [N].

Since $d(x) \leq d(y)+|x-y|$ one may observe that the condition $|x-y|<$ $\delta_{n} \max \{d(x), d(y)\}$ implies that also $|x-y|<\frac{\delta_{n}}{1-\delta_{n}} \min \{d(x), d(y)\}$.

The preceding theorem shows that the negative part of the Green function is uniformly bounded and hence relatively small when compared with the singular positive part, as long as $x$ or $y$ stays uniformly away from the boundary $\partial \Omega$.

Combining Theorem 1 with Green function estimates due to Krasovskij [K] and refined by Dall'Acqua and the second author [DS1], one obtains: 
Corollary 2. Under the assumptions of Theorem 1 there exists a constant $C=$ $C(\Omega)$ such that if $|x-y|<\delta_{n} \max \{d(x), d(y)\}$, then

$$
0<G_{\Omega}(x, y) \leq C \begin{cases}|x-y|^{4-n} & \text { for } n>4, \\ \log \left(1+\frac{1}{|x-y|}\right) & \text { for } n=4\end{cases}
$$

Remark 2.1. Except for the results of Nehari and the few explicit formulas for special domains, the estimates that we are aware of did not identify regions of positivity outside the diagonal. One knows that when $n \geq 4$ the Green function has a positive singularity, that is, $G_{\Omega}(x, y) \rightarrow+\infty$ for $x \rightarrow y$. The estimates that have been proved before in DS1, for general domains are

$$
\left|G_{\Omega}(x, y)\right| \leq C \begin{cases}|x-y|^{4-n} \min \left\{1,\left(\frac{d(x) d(y)}{|x-y|^{2}}\right)^{2}\right\} & \text { for } n>4 \\ \log \left(1+\left(\frac{d(x) d(y)}{|x-y|^{2}}\right)^{2}\right) & \text { for } n=4, \\ (d(x) d(y))^{2-\frac{1}{2} n} \min \left\{1,\left(\frac{d(x) d(y)}{|x-y|^{2}}\right)^{\frac{1}{2} n}\right\} & \text { for } n<4 .\end{cases}
$$

The estimates in (2) are of optimal order for the positive part of the Green function as can be seen by the explicit function from [B for the ball. See also GS2]. We expect that for the negative part much better estimates hold true, but except for special domains where an explicit formula for the Green function is known, only in two dimensions such a better estimate has been obtained [DMS]. For general two-dimensional smooth domains the following estimate is of optimal order:

$$
-C d(x)^{2} d(y)^{2} \leq G_{\Omega}(x, y) \leq C d(x) d(y) \min \left\{1, \frac{d(x) d(y)}{|x-y|^{2}}\right\} .
$$

With slightly more complicated but similar techniques as in the proof of Theorem 1, one may also cover the Green function for the Dirichlet problem $G_{(-\Delta)^{m}, \Omega}$ for the polyharmonic operator. By means of the formula

$$
u(x)=\int_{\Omega} G_{(-\Delta)^{m}, \Omega}(x, y) f(y) d y
$$

we find solutions of the polyharmonic Dirichlet problem

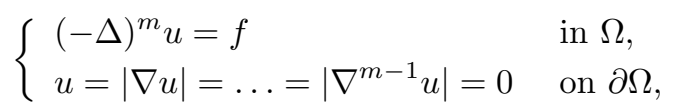

provided $f$ and $\Omega$ are smooth enough.

In order to avoid distinctions and too many technicalities, we only state and prove the result for large dimensions. Moreover, we think that, as in the biharmonic case [N], it cannot be extended to the whole range of small dimensions.

Theorem 3. Let $m \in \mathbb{N}, n>2 m$. Then there exists a constant $\delta_{m, n}>0$, which depends only on the dimension $n$ and the order $2 m$ of the polyharmonic operator, such that the following holds true.

Assume $\Omega \subset \mathbb{R}^{n}$ to be a $C^{2 m, \alpha}$-smooth bounded domain and let $G_{(-\Delta)^{m}, \Omega}$ denote the polyharmonic Green function under Dirichlet boundary conditions. If

$$
|x-y|<\delta_{m, n} \max \{d(x), d(y)\},
$$

then

$$
G_{(-\Delta)^{m}, \Omega}(x, y)>0
$$


For the constant $\delta_{m, n}$, one may achieve that

$$
\delta_{m, n=2 m+1} \geq 1+\frac{\Gamma(m) \Gamma\left(\frac{3}{2}\right)}{\Gamma\left(m+\frac{1}{2}\right)}-\sqrt{1+\frac{\Gamma(m)^{2} \Gamma\left(\frac{3}{2}\right)^{2}}{\Gamma\left(m+\frac{1}{2}\right)^{2}}}
$$

and, for fixed $m$, that

$$
\lim _{n \rightarrow \infty} \delta_{m, n}=\frac{\sqrt{5}-1}{2} \approx 0.618 .
$$

Remark 3.1. Numerical evidence indicates the following for the constants $\delta_{m, n}$.

- For each $m$ the sequence $\left\{\delta_{m, n}\right\}_{n=2 m+1}^{\infty}$ is increasing to $\frac{\sqrt{5}-1}{2}$.

- The sequence $\left\{\delta_{m, 2 m+1}\right\}_{m=2}^{\infty}$ is decreasing to 0 .

We emphasise that we provide bounds for $\delta_{m, n}$, the limit of which for $n \rightarrow \infty$ is $\frac{\sqrt{5}-1}{2}$ for each $m$.

\section{THE BIHARMONIC OPERATOR}

We consider the situation

$$
B_{1}:=B_{1}(0) \subset \Omega \subset B_{R}:=B_{R}(0)
$$

and write for suitable $f: \mathbb{R}^{n} \rightarrow \mathbb{R}$ :

$$
\mathcal{G}_{\Omega} f(x):=\int_{\Omega} G_{\Omega}(x, y) f(y) d y
$$

the solution $u(x):=\mathcal{G}_{\Omega} f(x)$ to the Dirichlet problem (1).

Let us recall a fundamental solution for $\Delta^{2}$ on $\mathbb{R}^{n}$ :

$$
F_{n}(|x|)= \begin{cases}c_{n}|x|^{4-n} & \text { if } n \notin\{2,4\}, \\ -2 c_{4} \log |x| & \text { if } n=4, \\ 2 c_{2}|x|^{2} \log |x| & \text { if } n=2,\end{cases}
$$

where

$$
c_{n}=\left\{\begin{array}{ll}
\frac{1}{2(n-4)(n-2) n e_{n}}, & \text { if } n \notin\{2,4\}, \\
\frac{1}{8 n e_{n}}, & \text { if } n \in\{2,4\},
\end{array} \quad e_{n}=\int_{B_{1}(0)} d x .\right.
$$

The Green function may be decomposed into the fundamental solution plus a regular part

$$
G_{\Omega}(x, y)=F_{n}(|x-y|)+H_{\Omega}(x, y),
$$

where $H_{\Omega} \in C^{4, \alpha}(\Omega \times \bar{\Omega})$. We will also use

$$
\mathcal{H}_{\Omega} f(x):=\int_{\Omega} H_{\Omega}(x, y) f(y) d y .
$$

Lemma 4. Let $f, g$ be smooth and supported in $B_{1}$. Then

$$
\begin{aligned}
4 \int_{\Omega}\left(\Delta \mathcal{G}_{\Omega} f\right)\left(\Delta \mathcal{G}_{\Omega} g\right) d x \geq & \int_{B_{1}}\left(f\left(\mathcal{H}_{B_{1}} f-\mathcal{H}_{B_{R}} f\right)+g\left(\mathcal{H}_{B_{1}} g-\mathcal{H}_{B_{R}} g\right)\right) d x \\
& +\int_{B_{1}}\left(f\left(\mathcal{G}_{B_{1}} g+\mathcal{G}_{B_{R}} g\right)+g\left(\mathcal{G}_{B_{1}} f+\mathcal{G}_{B_{R}} f\right)\right) d x
\end{aligned}
$$


Proof. We consider the quadratic form

$$
\mathbb{R}^{2} \ni(\beta, \gamma) \mapsto \int_{\Omega}\left(\beta \Delta \mathcal{G}_{\Omega} f+\gamma \Delta \mathcal{G}_{\Omega} g\right)^{2} d x
$$

and show that this is non-decreasing in the domain $\Omega$. For this purpose, consider smooth domains $\omega \subset \Omega$, and one gets that

$$
\begin{aligned}
& \int_{\Omega}\left(\beta \Delta \mathcal{G}_{\Omega} f+\gamma \Delta \mathcal{G}_{\Omega} g\right)^{2} d x-\int_{\omega}\left(\beta \Delta \mathcal{G}_{\omega} f+\gamma \Delta \mathcal{G}_{\omega} g\right)^{2} d x \\
&= \int_{\Omega}\left(\beta \Delta \mathcal{G}_{\Omega} f+\gamma \Delta \mathcal{G}_{\Omega} g\right)^{2} d x+\int_{\omega}\left(\beta \Delta \mathcal{G}_{\omega} f+\gamma \Delta \mathcal{G}_{\omega} g\right)^{2} d x \\
&-2 \int_{\omega}\left(\beta \mathcal{G}_{\omega} f+\gamma \mathcal{G}_{\omega} g\right)\left(\beta \Delta^{2} \mathcal{G}_{\omega} f+\gamma \Delta^{2} \mathcal{G}_{\omega} g\right) d x \\
&= \int_{\Omega}\left(\beta \Delta \mathcal{G}_{\Omega} f+\gamma \Delta \mathcal{G}_{\Omega} g\right)^{2} d x+\int_{\omega}\left(\beta \Delta \mathcal{G}_{\omega} f+\gamma \Delta \mathcal{G}_{\omega} g\right)^{2} d x \\
&-2 \int_{\omega}\left(\beta \mathcal{G}_{\omega} f+\gamma \mathcal{G}_{\omega} g\right)(\beta f+\gamma g) d x \\
&= \int_{\Omega}\left(\beta \Delta \mathcal{G}_{\Omega} f+\gamma \Delta \mathcal{G}_{\Omega} g\right)^{2} d x+\int_{\omega}\left(\beta \Delta \mathcal{G}_{\omega} f+\gamma \Delta \mathcal{G}_{\omega} g\right)^{2} d x \\
&-2 \int_{\omega}\left(\beta \mathcal{G}_{\omega} f+\gamma \mathcal{G}_{\omega} g\right)\left(\beta \Delta^{2} \mathcal{G}_{\Omega} f+\gamma \Delta^{2} \mathcal{G}_{\Omega} g\right) d x \\
&= \int_{\Omega}\left(\beta \Delta \mathcal{G}_{\Omega} f+\gamma \Delta \mathcal{G}_{\Omega} g\right)^{2} d x+\int_{\omega}\left(\beta \Delta \mathcal{G}_{\omega} f+\gamma \Delta \mathcal{G}_{\omega} g\right)^{2} d x \\
&-2 \int_{\omega}\left(\beta \Delta \mathcal{G}_{\omega} f+\gamma \Delta \mathcal{G}_{\omega} g\right)\left(\beta \Delta \mathcal{G}_{\Omega} f+\gamma \Delta \mathcal{G}_{\Omega} g\right) d x \\
&= \int_{\Omega \backslash \omega}\left(\beta \Delta \mathcal{G}_{\Omega} f+\gamma \Delta \mathcal{G}_{\Omega} g\right)^{2} d x \\
&+\int_{\omega}\left(\beta\left(\Delta \mathcal{G}_{\Omega} f-\Delta \mathcal{G}_{\omega} f\right)+\gamma\left(\Delta \mathcal{G}_{\Omega} g-\Delta \mathcal{G}_{\omega} g\right)\right)^{2} d x \\
& \geq 0 .
\end{aligned}
$$

In a first step we exploit this monotonicity in $B_{1} \subset \Omega$ with $\beta=\gamma=1$ :

$$
\int_{\Omega}\left(\Delta \mathcal{G}_{\Omega} f+\Delta \mathcal{G}_{\Omega} g\right)^{2} d x \geq \int_{B_{1}}\left(\Delta \mathcal{G}_{B_{1}} f+\Delta \mathcal{G}_{B_{1}} g\right)^{2} d x=\int_{B_{1}}(f+g) \mathcal{G}_{B_{1}}(f+g) d x .
$$

In a second step it is used in $\Omega \subset B_{R}$ with $\beta=-\gamma=1$ :

$$
\begin{aligned}
& \int_{\Omega}\left(\Delta \mathcal{G}_{\Omega} f-\Delta \mathcal{G}_{\Omega} g\right)^{2} d x \leq \int_{B_{R}}\left(\Delta \mathcal{G}_{B_{R}} f-\Delta \mathcal{G}_{B_{R}} g\right)^{2} d x \\
& =\int_{B_{R}}(f-g) \mathcal{G}_{B_{R}}(f-g) d x=\int_{B_{1}}(f-g) \mathcal{G}_{B_{R}}(f-g) d x .
\end{aligned}
$$

The first identity follows from an integration by parts and the boundary conditions for $G_{B_{R}}$, and the second since the support of $f$ and $g$ is supposed to lie in $B_{1}$. 
Substracting (9) from (8) yields

$$
\begin{aligned}
4 \int_{\Omega}\left(\Delta \mathcal{G}_{\Omega} f\right)\left(\Delta \mathcal{G}_{\Omega} g\right) d x & \geq \int_{B_{1}} f\left(\mathcal{G}_{B_{1}} f-\mathcal{G}_{B_{R}} f\right) d x+\int_{B_{1}} g\left(\mathcal{G}_{B_{1}} g-\mathcal{G}_{B_{R}} g\right) d x \\
& +\int_{B_{1}} f\left(\mathcal{G}_{B_{1}} g+\mathcal{G}_{B_{R}} g\right) d x+\int_{B_{1}} g\left(\mathcal{G}_{B_{1}} f+\mathcal{G}_{B_{R}} f\right) d x
\end{aligned}
$$

Since $\mathcal{G}_{B_{1}}-\mathcal{G}_{B_{R}}=\mathcal{H}_{B_{1}}-\mathcal{H}_{B_{R}}$, the claim follows.

Lemma 5. For $x, y \in B_{1}, x \neq y$, we have the following estimate from below for the biharmonic Green function of $\Omega$ :

$$
\begin{aligned}
G_{\Omega}(x, y) \geq & \frac{1}{4}\left(H_{B_{1}}(x, x)-H_{B_{R}}(x, x)+H_{B_{1}}(y, y)-H_{B_{R}}(y, y)\right) \\
& +\frac{1}{2}\left(G_{B_{1}}(x, y)+G_{B_{R}}(x, y)\right)
\end{aligned}
$$

Proof. The statement follows directly from Lemma 4 by taking smooth approximations of the Dirac delta distribution concentrated in $x$ and $y$ resp. for $f$ and $g$. One also uses the symmetry of the Green function: $G_{\Omega}(x, y)=G_{\Omega}(y, x)$.

Proof of Theorem 1. We recall (see e.g. [B, p. 126], cf. also [GS2, p. 591]) that for $n>4$

$$
\begin{aligned}
G_{B_{1}}(x, y) & =c_{n}\left\{|x-y|^{4-n}-\frac{n-2}{2}|| x\left|y-\frac{x}{|x|}\right|^{4-n}\right. \\
& \left.+\frac{n-4}{2}|| x\left|y-\frac{x}{|x|}\right|^{2-n}|x-y|^{2}\right\} \\
G_{B_{R}}(x, y) & =R^{4-n} G_{B_{1}}\left(\frac{1}{R} x, \frac{1}{R} y\right) \\
H_{B_{R}}(x, x) & =-c_{n} \frac{n-2}{2}\left(R-\frac{|x|^{2}}{R}\right)^{4-n}
\end{aligned}
$$

while for $n=4$

$$
\begin{aligned}
G_{B_{1}}(x, y) & =c_{4}\left\{-2 \log |x-y|+2 \log || x\left|y-\frac{x}{|x|}\right|\right. \\
\left.-1+|| x\left|y-\frac{x}{|x|}\right|^{-2}|x-y|^{2}\right\} & \\
G_{B_{R}}(x, y) & =G_{B_{1}}\left(\frac{1}{R} x, \frac{1}{R} y\right) \\
H_{B_{R}}(x, x) & =2 c_{4} \log \left(1-\frac{|x|^{2}}{R^{2}}\right)-c_{4}+2 c_{4} \log R
\end{aligned}
$$

In order to prove Theorem 1, it is enough, by scaling and translation, to consider $x=0, y \in B_{\delta_{n}}(0)$, where $\delta_{n} \in(0,1)$ has to be suitably specified below. 
We consider first the case $n>4$, where Lemma 5 and formulas (11)-(13) yield:

$$
\begin{aligned}
\frac{4}{c_{n}} G_{\Omega}(0, y) \geq & -\frac{n-2}{2}+\frac{n-2}{2} R^{4-n}-\frac{n-2}{2}\left(1-|y|^{2}\right)^{4-n} \\
& +\frac{n-2}{2}\left(R-\frac{|y|^{2}}{R}\right)^{4-n}+4|y|^{4-n}-(n-2) \\
& +(n-4)|y|^{2}-(n-2) R^{4-n}+(n-4) R^{2-n}|y|^{2} .
\end{aligned}
$$

Letting $R \rightarrow \infty$, we obtain

$$
\frac{4}{c_{n}} G_{\Omega}(0, y) \geq 4|y|^{4-n}+(n-4)|y|^{2}-\frac{n-2}{2}\left(1-|y|^{2}\right)^{4-n}-\frac{3}{2}(n-2) .
$$

If $n=5$ one has to check whether

$$
0<4-6|y|-4|y|^{2}+\frac{11}{2}|y|^{3}-|y|^{5} .
$$

The right-hand side is strictly decreasing in $|y| \in[0,0.6]$ and takes on a positive value for $|y|=0.6$. According to $\mathrm{MAPLE}^{\mathrm{TM}}$ the above inequality is satisfied for $|y| \in(0,0.612865 \ldots)$.

If $n \geq 6$, we drop the term $(n-4)|y|^{2}$ in (17) and have to determine $\delta_{n}$ such that

$$
4 \delta_{n}^{4-n}-\frac{n-2}{2}\left(1-\delta_{n}^{2}\right)^{4-n}-\frac{3}{2}(n-2) \geq 0 .
$$

Asymptotically, $\delta_{n}$ should be chosen close to the positive root $\delta_{\infty}$ of

$$
\delta=1-\delta^{2},
$$

i.e. to $\delta_{\infty}=(\sqrt{5}-1) / 2 \approx 0.618$. We show that (18) is satisfied with $\delta_{n}=0.6$, i.e. that

$$
\begin{aligned}
& 4\left(\frac{3}{5}\right)^{4-n}-\frac{n-2}{2}\left(\frac{16}{25}\right)^{4-n}-\frac{3}{2}(n-2) \geq 0 \\
\Leftrightarrow & 8-(n-2)\left(\frac{15}{16}\right)^{n-4}-3(n-2)\left(\frac{3}{5}\right)^{n-4} \geq 0 .
\end{aligned}
$$

The left-hand side of the last expression is increasing for $n \geq 18$ and attains positive values for $n=6, \ldots, 18$, thereby showing that (18) holds true for $\delta_{n}=0.6$.

Finally we consider the case $n=4$ and choose $R \geq 1$. Lemma 5 and formulas (14) -(16) yield:

$$
\begin{aligned}
\frac{4}{c_{4}} G_{\Omega}(0, y) \geq & -2 \log \left(1-\frac{|y|^{2}}{R^{2}}\right)+2 \log \left(1-|y|^{2}\right)-4 \log R-8 \log |y|+4 \log R \\
& -4+2|y|^{2}+2 \frac{|y|^{2}}{R^{2}} \\
\geq & -2 \log \left(1-\frac{|y|^{2}}{R^{2}}\right)+2 \log \left(1-|y|^{2}\right)-8 \log |y|-4+2|y|^{2}+2 \frac{|y|^{2}}{R^{2}} .
\end{aligned}
$$

Letting $R \rightarrow \infty$, we conclude that

$$
\frac{4}{c_{4}} G_{\Omega}(0, y) \geq-8 \log |y|+2 \log \left(1-|y|^{2}\right)-4+2|y|^{2} .
$$

The right-hand side is certainly decreasing in $|y| \in[0,0.6]$ and takes on a positive value for $|y|=\delta_{4}=0.59$. With the help of MAPLE ${ }^{\mathrm{TM}}$ we see that it is positive for $|y| \in(0,0.594160 \ldots)$. 


\section{THE POLYHARMONIC OPERATOR}

Here, the arguments are very similar to Section 2 and we may be very brief and focus mainly on what is different. Throughout this section, according to Theorem 3 , we confine ourselves to the case

$$
n>2 m \text {. }
$$

We consider

$$
B_{1}=B_{1}(0) \subset \Omega \subset B_{R}=B_{R}(0)
$$

and the Green function $G_{(-\Delta)^{m}, \Omega}$ corresponding to (3) in $\Omega$. Again, this Green function may be decomposed into a singular and a regular part

$$
G_{(-\Delta)^{m}, \Omega}(x, y)=c_{m, n}|x-y|^{2 m-n}+H_{(-\Delta)^{m}, \Omega}(x, y),
$$

where $H_{(-\Delta)^{m}, \Omega} \in C^{2 m, \alpha}(\Omega \times \bar{\Omega})$ denotes the regular part and $c_{m, n}>0$ is a suitable positive constant. Lemma 5 directly generalises to the polyharmonic situation and we may perform the

Proof of Theorem [3. According to [B, p. 126] (see also [GS2, p. 591]) we have with a suitable positive constant $k_{m, n}$ :

$$
\begin{aligned}
& G_{(-\Delta)^{m}, B_{1}}(x, y)=k_{m, n}|x-y|^{2 m-n} \int_{1}^{|| x\left|y-\frac{x}{|x|}\right| /|x-y|}\left(v^{2}-1\right)^{m-1} v^{1-n} d v \\
& G_{(-\Delta)^{m}, B_{R}}(x, y)=R^{2 m-n} G_{(-\Delta)^{m}, B_{1}}\left(\frac{1}{R} x, \frac{1}{R} y\right), \\
& H_{(-\Delta)^{m}, B_{R}}(x, x)=-\frac{k_{m, n}}{n-2 m}\left(R-\frac{|x|^{2}}{R}\right)^{2 m-n} .
\end{aligned}
$$

The constants $C_{m, n}$ and $k_{m, n}$ are related by

$$
\begin{aligned}
c_{m, n} & =k_{m, n} \int_{1}^{\infty}\left(v^{2}-1\right)^{m-1} v^{1-n} d v=k_{m, n}(-1)^{m} \sum_{j=0}^{m-1} \frac{(-1)^{j}\left(\begin{array}{c}
m-1 \\
j
\end{array}\right)}{2 j+2-n} \\
& =k_{m, n} \frac{2^{m-1}(m-1) !}{\prod_{j=1}^{m}(n-2 j)},
\end{aligned}
$$

the proof of which is a calculus exercise.

By the generalisation of Lemma [5, formulas (21)-(23), and letting $R \rightarrow \infty$, we obtain

$$
\begin{aligned}
G_{(-\Delta)^{m}, \Omega}(0, y) & \geq c_{m, n}|y|^{2 m-n}-\frac{k_{m, n}}{2}|y|^{2 m-n} \int_{1 /|y|}^{\infty}\left(v^{2}-1\right)^{m-1} v^{1-n} d v \\
& -\frac{k_{m, n}}{4(n-2 m)}\left(1+\left(1-|y|^{2}\right)^{2 m-n}\right) \\
& \geq \frac{c_{m, n}}{2}|y|^{2 m-n}-\frac{k_{m, n}}{4(n-2 m)}\left(1+\left(1-|y|^{2}\right)^{2 m-n}\right)
\end{aligned}
$$

so that

$$
\frac{4(n-2 m)}{k_{m, n}} G_{(-\Delta)^{m}, \Omega}(0, y) \geq \frac{2(m-1) !}{\prod_{j=1}^{m-1}\left(\frac{n}{2}-j\right)}|y|^{2 m-n}-1-\left(1-|y|^{2}\right)^{2 m-n} .
$$


Certainly, one finds $\delta_{m, n}>0$ such that the right-hand side is positive for $|y|<\delta_{m, n}$. For $m$ fixed and $n \rightarrow \infty$, the powers $2 m-n$ dominate all the other terms and $\delta_{m, n}$ may be chosen such that they approach the positive zero $\delta_{\infty}$ of

$$
\delta=1-\delta^{2},
$$

which is presicely $\frac{\sqrt{5}-1}{2}$. In the case $n=2 m+1$, (26) reads

$$
\begin{aligned}
\frac{4}{k_{m, 2 m+1}} G_{(-\Delta)^{m}, \Omega}(0, y) & \geq 2 \frac{\Gamma(m) \Gamma\left(\frac{3}{2}\right)}{\Gamma\left(m+\frac{1}{2}\right)} \cdot \frac{1}{|y|}-1-\frac{1}{1-|y|^{2}} \\
& \geq 2 \frac{\Gamma(m) \Gamma\left(\frac{3}{2}\right)}{\Gamma\left(m+\frac{1}{2}\right)} \cdot \frac{1}{|y|}-1-\frac{1}{1-|y|} .
\end{aligned}
$$

The right-hand side (28) is positive if and only if

$$
|y|<1+\frac{\Gamma(m) \Gamma\left(\frac{3}{2}\right)}{\Gamma\left(m+\frac{1}{2}\right)}-\sqrt{1+\frac{\Gamma(m)^{2} \Gamma\left(\frac{3}{2}\right)^{2}}{\Gamma\left(m+\frac{1}{2}\right)^{2}}} .
$$

One might wonder whether dropping a positive term in (25) gives rise to a very rough estimate. The previous estimate (29) would still allow for choosing $\delta_{2,5}=$ 0.46 , while the right-hand side of (27) is positive for $|y|<\delta_{2,5}=0.54$. On the other hand, according to Theorem $1, \delta_{2,5}=0.59$ is admissible. This shows that one hasn't lost much in (25). In any case, our proof shows that we cannot do better than a constant $\delta_{m, n}$ with

$$
\lim _{m \rightarrow \infty} \delta_{m, 2 m+1}=0
$$

even if one had kept the second term in (25).

\section{REFERENCES}

[ADN] S. Agmon, A. Douglis, L. Nirenberg, Estimates near the boundary for solutions of elliptic partial differential equations satisfying general boundary conditions. I, Commun. Pure Appl. Math. 12, 623-727 (1959). MR0125307(23:A2610)

[B] T. Boggio, Sulle funzioni di Green d'ordine m, Rend. Circ. Mat. Palermo 20, 97-135 (1905).

[C] C.V. Coffman, On the structure of solutions to $\Delta^{2} u=\lambda u$ which satisfy the clamped plate conditions on a right angle, SIAM J. Math. Anal. 13, 746-757 (1982). MR0668318 (84a:35015)

[CD] C.V. Coffman, R.J. Duffin, On the structure of biharmonic functions satisfying the clamped plate conditions on a right angle, Adv. Appl. Math. 1, 373-389 (1980). MR0603137 (82e:31004)

[CG] C.V. Coffman, C.L. Grover, Obtuse cones in Hilbert spaces and applications to partial differential equations, J. Funct. Anal. 35, 369-396 (1980). MR0563561 (81m:46038)

[DS1] A. Dall'Acqua, G. Sweers, Estimates for Green function and Poisson kernels of higher order Dirichlet boundary value problems, J. Differential Equations 205, 466-487 (2004). MR.2092867(2005i:35065)

[DS2] A. Dall'Acqua, G. Sweers, The clamped plate equation on the limaçon, Ann. Mat. Pura Appl. 184, 361-374 (2005). MR2164263 (2006i:35066)

[DMS] A. Dall'Acqua, Ch. Meister, G. Sweers, Separating positivity and regularity for fourth order Dirichlet problems in 2d-domains, Analysis 25, 205-261 (2005). MR2232852

[D] R.J. Duffin, On a question of Hadamard concerning super-biharmonic functions, J. Math. Phys. 27, 253-258 (1949). MR0029021 (10:534h)

[Ga] P.R. Garabedian, Partial Differential Equations, second edition, Chelsea: New York, 1986. MR.0943117 (89c:35001) 
[GS1] H.-Ch. Grunau, G. Sweers, Positivity for perturbations of polyharmonic operators with Dirichlet boundary conditions in two dimensions, Math. Nachr. 179, 89-102 (1996). MR.1389451 (97f:35040)

[GS2] H.-Ch. Grunau, G. Sweers, Positivity for equations involving polyharmonic operators with Dirichlet boundary conditions, Math. Ann. 307, 589-626 (1997). MR 1464133 (98g:35058)

[Ha1] J. Hadamard, Mémoire sur le problème d'analyse relatif à l'équilibre des plaques elastiques encastrées, in: (Euvres de Jacques Hadamard, Tome II, 515-641, CNRS Paris, 1968. MR0230598 (37:6158)

[Ha2] J. Hadamard, Sur certains cas intéressants du problème biharmonique, in: (Euvres de Jacques Hadamard, Tome III, 1297-1299, CNRS Paris, 1968. MR0230598 (37:6158)

[K] J.P. Krasovskij, Isolation of singularities of the Green's function (Russian), Izv. Akad. Nauk SSSR Ser. Mat. 31, 977-1010 (1967), English translation in Math. USSR, Izv. 1, 935-966 (1967). MR0223740 (36:6788)

[M] V.A. Malyshev, The Hadamard conjecture and estimates of the Green function (Russian), Algebra Anal. 4, 1-44 (1992), English translation in: St. Petersbg. Math. J. 4, 633-666 (1993). MR.1190781 (94c:35071)

[N] Z. Nehari, On the biharmonic Green's function, in Studies Math. Mech., presented to Richard von Mises, 111-117 (1954). MR0064992 (16:368f)

[O] St. Osher, On Green's function for the biharmonic equation in a right angle wedge, $J$. Math. Anal. Appl. 43, 705-716 (1973). MR0324209 (48:2561)

[S] E. Sassone, Positivity for polyharmonic problems on domains close to a disk, Ann. Mat. Pura Appl. 186, 419-432 (2007).

[ST] H.S. Shapiro, M. Tegmark, An elementary proof that the biharmonic Green function of an eccentric ellipse changes sign, SIAM Rev. 36, 99-101 (1994). MR1267051 (94m:35096)

FAkultät Für Mathematik, Otto-Von-Guericke-Universität, Postfach 4120, 39016 Magdeburg, Germany

E-mail address: Hans-Christoph.Grunau@mathematik.uni-magdeburg.de

Mathematisches Institut, Universität Zu Köln, Weyertal 86-90, 50931 Köln, Germany; and Delft Institute of Applied Mathematics, Delft University of Technology, PO Box 5031, 2600 GA Delft, The Netherlands

E-mail address: gsweers@math.uni-koeln.de

E-mail address: G.H.Sweers@tudelft.nl 